\title{
Persistent current in disordered Aharonov-Bohm rings with interacting electrons
}

\author{
Qianghua Wang \\ Department of Physics, University of Hong Kong, Pokfulam, Hong Kong \\ and Department of Physics, Nanjing University, Nanjing 210093, China \\ Z. D. Wang and Jian-Xin Zhu \\ Department of Physics, University of Hong Kong, Pokfulam, Hong Kong
}

(Received 22 March 1996; revised manuscript received 14 May 1996)

\begin{abstract}
The role of repulsive on-site and nearest-neighbor Coulomb interactions in disordered half-filled AharanovBohm rings is studied by world-line quantum Monte Carlo simulations. The diverse dependence of the equilibrium persistent current on the couplings is found to relate systematically to the magnetic phase of the model: the maximum charge stiffness (or the persistent current) coexists with the phase-transition line between the dominant charge-density-wave state and the dominant spin-density-wave state. The stiffness vanishes with an increasing departure from the transition line. Thus in the disordered rings the Coulomb interactions can enhance the charge stiffness over the noninteracting limit in such a way as to drive the system toward the phase-transition regime. [S0163-1829(96)01335-5]
\end{abstract}

\section{INTRODUCTION}

The role of Coulomb interactions between electrons in disordered metallic mesoscopic rings has been attracting much interest recently. The experiment performed on a ballistic ring $^{1}$ showed an equilibrium persistent current driven by an Aharonov-Bohm (AB) magnetic flux $\Phi=\oint \mathbf{A} \cdot d \mathbf{l}$ with a magnitude of the current in agreement with the prediction of Büttiker, Imry, and Landauer. ${ }^{2}$ However, two other experiments, ${ }^{3,4}$ believed to be in the diffusive regime, gave a magnitude of the current one to two orders larger than what would be expected from a simple theory of free electrons in disordered rings. It is natural to take Coulomb interactions into account in order to resolve such a discrepancy. In a weakly disordered one-dimensional (1D) spinless fermion model, it was found from the Hartree-Fock approximation that the Coulomb interaction always suppresses the persistent current. ${ }^{5}$ In the localized regime of the same model, however, the exact diagonalization calculations of Abraham and Berkovits show some enhancement of the current. ${ }^{6}$ More interestingly, a recent exact diagonalization study by Berkovits and Avishi revealed a strong enhancement of the current in the diffusive regime for $2 \mathrm{D}$ spinless systems. ${ }^{7}$ A similar effect was also found by the Hartree-Fock approximation for 2D (Ref. 8) and 3D (Ref. 9) spinless systems. The spinless model is relevant when the separation of the spin and charge degrees of freedom is fairly complete. Otherwise, one needs to consider the original disordered Hubbard model to study the interplay between the disorder and interactions. ${ }^{10-12}$ Recently, it was found from renormalization-group calculations of the Luttinger-liquid model that, with the spin degree of freedom included, the repulsive on-site interaction also enhances the current beyond a critical disorder strength over the noninteracting limit. ${ }^{10,11}$ Giamarchi and Shastry also discussed the extended Hubbard model with on-site and the nearest-neighbor interactions, respectively. ${ }^{11}$ They conjectured that the current (represented by their stiffness; see also below) would be enhanced by interactions whenever the charge-density fluctuation could be smeared.

It should be pointed out that all of the above theoretical and numerical studies gave a current magnitude in the diffusive regime that is still significantly lower than the clean limit for free electrons, even if the current could be enhanced by interactions over the noninteracting limit. On the other hand, the nearest-neighbor interaction was often ignored in renormalization-group studies. Since the latter is not at the disposal of experimentalists, and the interplay between various couplings is nontrivial on the theoretical side, we perform quantum Monte Carlo simulations for the extended disordered half-filled band Hubbard model, in which the reduction of the current by the disorder would be most severe in the absence of Coulomb interactions. ${ }^{10}$ The charge stiffness, as a measure of the magnitude of the persistent current, is calculated as a function of the Coulomb interactions at various disorder strengths. We find that at a fixed disorder strength the charge stiffness is a well-defined function of the departure from the phase boundary in the parameter space separating the charge-density-wave (CDW) dominant phase and the spin-density-wave (SDW) dominant phase, being suppressed deeply in both phases. According to this picture, Coulomb interactions enhance the current only if they help drive the system toward the phase-transition region. (The results in Ref. 11 turn out to be consistent with this picture.) The distinctively different behaviors of interactions in the weak and strong disorder limits are also discussed. The structure of this paper is as follows. After an outline of the model and the simulation method in Sec. II, the simulation results are presented and discussed in Sec. III. Finally, Sec. IV contains a summary of this work.

\section{MODEL AND SIMULATION METHOD}

The extended repulsive Hubbard model on a ring of size $L$ threaded by an AB flux $\Phi$ is described by the Hamiltonian 


$$
\begin{aligned}
H= & -t \sum_{i, \sigma}\left(e^{i \theta} c_{i+1, \sigma}^{\dagger} c_{i, \sigma}+\text { H.c. }\right)+U \sum_{i} n_{i, \uparrow} n_{i, \downarrow} \\
& +V \sum_{i} n_{i} n_{i+1}+\sum_{i} \epsilon_{i} n_{i},
\end{aligned}
$$

where $\sigma=-\bar{\sigma}= \pm 1$ denotes spin-up and spin-down states; $c_{i, \sigma}^{\dagger}$ and $c_{i, \sigma}$ create and annihilate spin- $\sigma$ fermions on site $i ; n_{i, \sigma}=c_{i, \sigma}^{\dagger} c_{i, \sigma}$ is the density operator for spin- $\sigma$ fermions; $\theta=2 \pi \Phi / L \Phi_{0}$, with $\Phi_{0}=h / e$ being a flux quantum; $n_{i}=n_{i, \uparrow}+n_{i, \downarrow}$ is the total density operator at site $i$, and finally, $\epsilon_{i}$ is the quenched random site energy which is assumed to be uniformly distributed in the interval $[-W, W]$, where $W$ is a measure of the disorder strength. In the absence of disorder and in the half-filled band with zero magnetization (or with equal spin-up and -down fermions), a meanfield theory predicts a zero-temperature phase transition between the CDW and SDW phases at $U=2 \mathrm{~V}$. More elaborate quantum Monte Carlo simulations ${ }^{13}$ showed that the transition line is slightly above the mean-field transition line in favor of slightly larger $V$, and that the transition changes from second order to first order beyond a critical coupling $U_{c} \approx 3$ (henceforth we measure all energies in units of $t$ ). On the other hand, it was shown in Ref. 10 that the suppression of the current by the on-site energy $U$ in the absence of the nearest-neighbor energy $V$ is the most severe when the system is close to the half-filled band. Therefore it is interesting to note what the behavior will be with finite $V$ and finite disorder $W$.

Recently, based upon earlier world-line quantum Monte Carlo methods, ${ }^{14-16}$ we have developed a scheme to calculate the global phase dependence of the persistent current at a fixed temperature for fermions in one-dimensional rings. ${ }^{17}$ The reliability of this scheme has been established by checking it against rigorous known results for noninteracting spinless fermions. To be more precise, from a checkerboard decomposition of the canonical partition function $Z=\operatorname{Tr} e^{-\beta H}$ (the checkerboard having a topology of a torus), the free energy $F$ can be expressed as a functional of the so-called (normalized) winding number distribution $P\left(w_{\uparrow}, w_{\downarrow}\right):{ }^{17}$

$$
\begin{aligned}
F= & -\frac{1}{\beta} \ln \widetilde{Z}-\frac{1}{\beta} \ln \sum_{w_{\uparrow}, w_{\downarrow}} P\left(w_{\uparrow}, w_{\downarrow}\right) \\
& \times \exp \left\{i \sum_{\sigma} w_{\sigma}\left[\varphi+\left(M_{\sigma}-1\right) \pi\right]\right\},
\end{aligned}
$$

where $\widetilde{Z}$ is an auxiliary partition function with elements being positive-definite and independent of the $\mathrm{AB}$ phase, ${ }^{18}$ $\varphi=2 \pi \Phi / \Phi_{0}$ is the reduced phase, and $M_{\sigma}$ and $w_{\sigma}$ $(=0, \pm 1, \pm 2, \ldots)$ are the particle number and the winding number contributed by the $\sigma$-spin fermions, respectively. The winding number distribution is sampled according to $\widetilde{Z}$ instead of $Z$ itself (which might include complex weighting terms in the checkerboard decomposition scheme in the presence of the $\mathrm{AB}$ phase), also being independent of the magnetic field. ${ }^{15,17}$ The response of the free energy to a finite Aharonov-Bohm flux is a measure of the equilibrium persistent current given by $J=-(e / \hbar)(\partial / \partial \varphi) F(\varphi)$.
Equation (2) is a straightforward generalization of the formula derived for the spin-less fermions in Ref. 17 and an analogous formula for bosons in one-dimensional rings. ${ }^{15} \mathrm{It}$ also bears a close resemblance to the expression in the framework of a one-dimensional Luttinger-liquid theory (cf. Ref. 19). It should be pointed out that while we always have $P\left(-w_{\uparrow},-w_{\downarrow}\right)=P\left(w_{\uparrow}, w_{\downarrow}\right)$, the symmetry $P\left(w_{\sigma},-w_{\bar{\sigma}}\right)$ $=P\left(w_{\sigma}, w_{\bar{\sigma}}\right)$ is broken in the presence of Coulomb coupling. This means that $P\left(w_{\uparrow}, w_{\downarrow}\right)$ can only be factorized as $P_{\uparrow}\left(w_{\uparrow}\right) P_{\downarrow}\left(w_{\downarrow}\right)$ for free fermions. In general, there will be significant sign cancellation for arbitrary $M_{\sigma}$ and $\varphi$ in the summation of Eq. (2), which imposes too stringent an accuracy requirement on the computation of the winding number distribution. However, the situation is simplified in the zeromagnetization sector $M_{\uparrow}=M_{\downarrow}$ (or in any other sector where $M_{\uparrow}$ and $M_{\downarrow}$ enjoy the same parity; however, following convention $^{10}$ we shall limit ourselves to the zeromagnetization sector). The free energy is minimal at $\varphi=\varphi_{c}=2 n \pi$ (or $\left.2 n \pi+\pi\right)$ when $M_{\uparrow}$ is odd (or even). At $\varphi_{c}$ we can define a charge stiffness as

$$
D_{c}=\left.\frac{L}{2} \frac{\partial^{2}}{\partial \varphi^{2}} F\right|_{\varphi=\varphi_{c}} \propto\left\langle\left(w_{\uparrow}+w_{\downarrow}\right)^{2}\right\rangle \equiv\left\langle w^{2}\right\rangle,
$$

where the second proportion follows from a Taylor expansion of Eq. (2) near $\varphi_{c}$. The charge stiffness provides an operational definition for the persistent current for small values of flux change $\delta \varphi(|\delta \varphi|<\pi)$ with respect to $\varphi_{c}$, given as $J=2 D_{c} \delta \varphi$ (in units of $I_{0} \equiv e t / L \hbar$ ). Although knowledge of the winding number distribution provides the global phase dependence of the persistent current ${ }^{17}$ as well as the charge stiffness, the computation is too expensive to change the topological winding number of the checkerboard configuration if the size of the lattice is not small. Instead, to determine $D_{c}$ we borrow a page from Ref. 15 , so that we only need to work in the zero winding number sector $\left(w_{\sigma}=0\right)$. Consider the pseudocurrent

$$
\mathcal{J}(\tau)=\sum_{i, \sigma} R_{i}\left[n_{i, \sigma}(\tau+\Delta \tau)-n_{i, \sigma}(\tau)\right]
$$

where $R_{i}$ is the space coordinate of the $i$ th site, $\tau$ is the imaginary time along the Trotter direction of the checkerboard, and a periodic condition in the space direction is implied. Equation (4) measures the clockwise displacement of the center-of-mass of the fermions during one imaginarytime step. Then the average $\left\langle w^{2}\right\rangle$ is proportional to the extrapolated zero-frequency limit of the Fourier transform of the "current-current" correlation function $C(\tau)=\langle\mathcal{J}(0) \mathcal{J}(\tau)\rangle .^{20}$

To check the above simplified algorithm for the stiffness, one should in principle compare the results with those of the exact diagonalization. Since the latter results are presently not available to us for extended Hubbard systems, we would rather compare the Monte Carlo results with rigorous results for clean and small systems with free electrons. This should be sufficient to show whether the simplified algorithm works. We note that the same algorithm has long been used to evaluate the superfluid density in bose systems (see, e.g., 
TABLE I. Charge stiffness vs the inverse temperature in a foursite ring with four electrons. $D_{c}$ and $D_{c}^{\mathrm{MC}}$ are the rigorous stiffness and the Monte Carlo stiffness, respectively.

\begin{tabular}{lcccc}
\hline \hline$\beta$ & 2 & 3 & 4 & 8 \\
\hline$D_{c}$ & 0.6345 & 0.7010 & 0.7066 & 0.7071 \\
$D_{c}^{\mathrm{MC}}$ & $0.636 \pm .04$ & $0.670 \pm 0.03$ & $0.699 \pm 0.03$ & $0.701 \pm 0.03$ \\
\hline \hline
\end{tabular}

Ref. 15). Table I shows the stiffness versus inverse temperature in a four-site half-filled ring with free electrons, where $D_{c}$ and $D_{c}^{\mathrm{MC}}$ are the rigorous stiffness and the Monte Carlo stiffness from the correlation function $C(\tau)$ limited to the zero-winding-number sector, respectively. Clearly, the Monte Carlo results methods agree with the rigorous results. The statistical error is lower than the errors given in the table, which represent the uncertainties of the zero-frequency extrapolation. We used imaginary time steps $\Delta \tau=\frac{1}{8}$ and $\Delta \tau=\frac{1}{4}$ in the Monte Carlo simulations, respectively, but found no discrepancy larger than the extrapolation uncertainty.

To obtain a rough idea of the magnetic phase of the system, we shall also need the CDW structure factor

$$
S(q)=\frac{1}{L} \sum_{i, j} \exp \left[i q\left(R_{i}-R_{j}\right)\right]\left\langle n_{i} n_{j}\right\rangle
$$

and the zero-frequency SDW susceptibility

$$
\begin{aligned}
\chi(q)= & \frac{1}{L} \int_{0}^{\beta} d \tau \sum_{i, j} \exp \left[i q\left(R_{i}-R_{j}\right)\right]\left\langle\left[n_{i, \uparrow}(\tau)-n_{i, \downarrow}(\tau)\right]\right. \\
& \left.\times\left[n_{j, \uparrow}(0)-n_{j, \downarrow}(0)\right]\right\rangle .
\end{aligned}
$$

If we scale the spatial size $L$ and the inverse temperature $\beta$ by the same factor, $S(q=\pi)$ will diverge linearly with $L$ if we are in the CDW phase characterized by the predominant double occupation of fermions at every other site, and $\chi(q=\pi)$ will diverge linearly in the SDW phase characterized by the predominant single occupation of the fermions at each site. By comparing $S(\pi)$ and $\chi(\pi)$ as functions of the parameters, we can determine the transition line qualitatively. Of course, to determine the transition line more accurately, one should study the scaling behavior of the correlation functions versus different lattice sizes. However, we shall see that, combining with the correlation functions, one can readily determine the transition line in finite systems by investigating the distinctive behavior of $D_{c}$, without going into scaling analysis, which is too expensive for us.

The quantum Monte Carlo simulations give the finitetemperature charge stiffness. In order to probe the groundstate property we have to go into inverse temperatures, scaling linearly with the size $L$ of the ring. We use $\beta=L$ in the following simulations. That the results represent the zerotemperature limit is checked by halving and doubling the inverse temperature. Our experience showed that $\beta=L$ gives an already reliable ground-state limit at least for the present purposes (cf. Ref. 13).

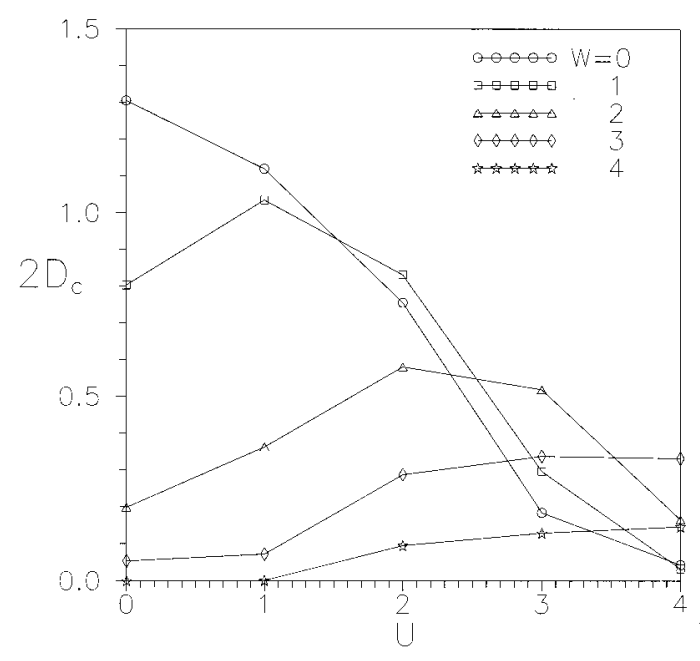

FIG. 1. The charge stiffness vs the on-site $U$ in the absence of the nearest-neighbor $V$ at various levels of disorders, $W=0$ (circles), $W=1$ (squares), $W=2$ (triangles), $W=3$ (diamonds), and $W=4$ (stars). Lines are drawn for a guide to the eye.

\section{SIMULATION RESULTS AND DISCUSSIONS}

In this section we present the charge stiffness $\left(D_{c}\right)$ versus the Coulomb interactions ( $U$ and $V$ ) and the disorder strength $(W)$ in rings of size $L=8$ in the half-filled band and zero-magnetization sector. While the results for larger rings are briefly discussed at the end of this section. Up to 20 realizations of the quenched disorders have been used to perform the disorder averages. The errors of the data in the following, obtained by dividing the 20 disorder ensembles into four groups, are within the corresponding symbol size. The systematic error of the zero-frequency extrapolation for $D_{c}$ is fairly less than 0.1 , and is not explicitly shown in the following figures.

Figure 1 shows the $U$ dependence of $D_{c}$ at various $W$ in the absence of the nearest-neighbor interaction, $(V=0)$. There is an evident monotonic reduction of $D_{c}$ with increasing $U$ in the clean ring, in contrast to the irrelevance of the interaction, as one would expect in an infinite system due to the Galilean invariance. This is understandable from the fact that even in the clean lattice model, the center-of-mass momentum is conserved only up to the reciprocal-lattice vector. With the inclusion of impurities, there exists a critical on-site coupling $U_{c}(V=0, W)$ beyond which the charge stiffness is enhanced by the disorder over the clean limit. Furthermore, at a fixed disorder strength we see that $D_{c}$ develops a maximum with increasing $U$ from zero, and the maximum becomes broader for stronger disorders. In Ref. 10, from renormalization-group calculations the authors were able to identify a critical disorder strength $W_{c}(V=0)$ beyond which the on-site interaction always enhances the charge stiffness over the noninteracting stiffness (cf. Ref. 11). (Note that in their work the increase of the effective disorder is reflected by enlargement of the system size at fixed bare disorder strength, which is not plausible for us in the Monte Carlo simulations due to limit computation resource.) The curve at $W=4$ in Fig. 1 clearly shows such a tendency, as it increases monotonically in the parameter range we considered. However, we are unable to go into stronger disorders since the 


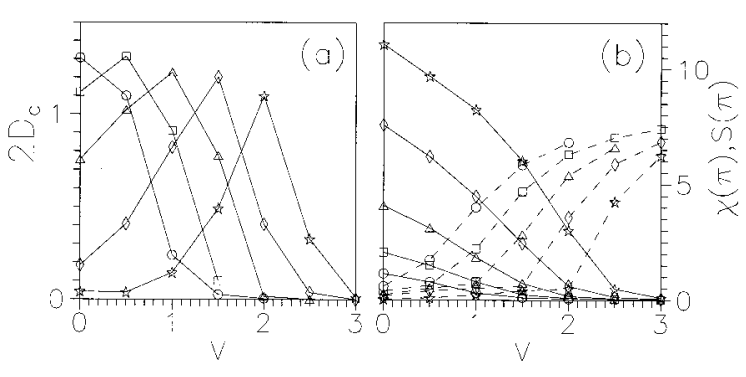

FIG. 2. (a) The charge stiffness vs $V$ at various values of the on-site repulsion, $U=0$ (circles), $U=1$ (squares), $U=2$ (triangles), $U=3$ (diamonds), and $U=4$ (stars). The disorder strength is fixed at $W=0$. (b) The corresponding CDW structure factor $S(\pi)$ (dashed line connected) and the zero-frequency SDW susceptibility $\chi(\pi)$ (solid line connected).

charge stiffness would be lower than our numerical resolution (in the zero-frequency extrapolation).

Figure 2(a) shows the charge stiffness versus the nearestneighbor interaction $V$ at fixed $U$ in clean rings $(W=0)$. Obviously $D_{c}$ develops a peak at some $V=V_{c}(W=0, U)$, beyond which $D_{c}$ is suppressed by $V$. Figure 2(b) shows the corresponding CDW structure factor $S(\pi)$ (dashed line connected) and the zero-frequency SDW susceptibility $\chi(\pi)$ (solid line connected). We observe that $V_{c}(W=0, U) \sim U / 2$ consistently follows the on-set value of $V$ at which $S(\pi)$ $[\chi(\pi)]$ begins to increase (decrease) sharply with increasing $V$ from $V<U / 2$. This strongly signals that the maximum charge stiffness coexists with the phase transition line. According to this picture, the transition point of $V$ at large $U$ can be estimated from Fig. 2(a) to be larger than the meanfield value $V=U / 2$ (e.g., $2<V<2.5$ at $U=4$ ), in accordance with the elaborate results of Hirsch. ${ }^{13}$ Thus our simplified determinations of the transition line is justified for qualitative purposes.

In the presence of disorders, there will be no long-rangeordered magnetic phases, as the CDW can be pinned by the disorders to form a pinned CDW phase. However, the distinct behavior of the stiffness and the correlation functions can still be used to quantify the dominant correlation in the disordered systems. Figures $3-5$ are the same plots as Fig. 2 except that the disorders are included as $W=1,2$, and 3 , respectively. We see the same qualitative behavior of the charge stiffness versus $V$, except that at the same level of $U, V_{c}(W, U)$ shifts to smaller values with increasing disorder, and there is a significant reduction of the maximum value of $D_{c}$ at elevated disorders. (With increasing $W$ the peaks at lower $U$ disappear.) The nearest-neighbor interac-
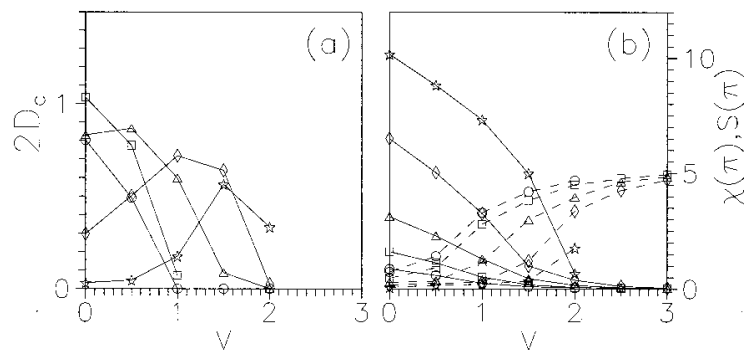

FIG. 3. The same plot as Fig. 2, except that $W=1$.

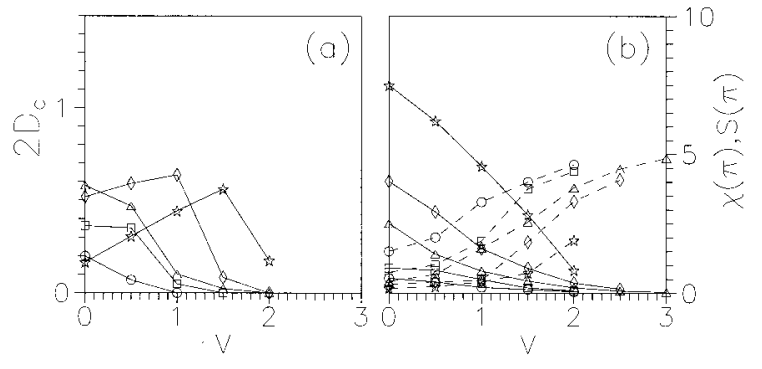

FIG. 4. The same plot as Fig. 2, except that $W=2$.

tion is seen to enhance (suppress) the CDW (SDW) correlations in all cases. On the other hand, the CDW correlations at $V=0$ increases steadily with increasing disorders, but $U$ renders the $S(\pi)$ smaller at each level of disorders. Therefore it is likely that the CDW correlation may be dominant at finite but small $U$ even at $V=0$ in the presence of strong disorders. Switching on $V$ would drive the system deeper into the pinned CDW phase. This explains why there are no peaks at small $U$ in the left panels of Figs. 3-5.

From the body of data in the above figures and the peaks for $D_{c}$, we are able to draw a schematic phase-transition line (due to the limited numerical resolution available) in the $V$ $U$ parameter space at each level of disorders in Fig. 6. Below (above) the transition line, the system is in the SDW (CDW) dominant phase. The transition line shifts towards the rightbottom side of the $V-U$ parameter space and intersects with the $V=0$ axis at elevated $U_{c}(W, V=0)$ with increasing disorder. This is understandable from the fact that while the repulsive on-site coupling competes with the disorder, the nearest-neighbor coupling helps the fermions to form a pinned CDW state. At $V=0$, the competition between the disorder and the on-site coupling should come into balance at roughly $U_{c}(W, V=0) \sim\left\langle\epsilon_{i}^{2}\right\rangle^{1 / 2}=W / \sqrt{3}$.

Collecting the above results, we conclude that at a fixed disorder strength, the charge stiffness will be enhanced by the Coulomb interactions which help drive the system toward the phase-transition regime. Therefore, at large $U$ and moderate $W$, the charge stiffness will be lifted essentially by a small nearest-neighbor coupling $V$ (e.g., $V<U / 2)$, without which the system would be deep inside the SDW phase with a vanishing stiffness. The above picture is also consistent with the results in Fig. 1: at a finite disorder (and with $V=0$ ), increasing $U$ from zero drives the system from the pinned CDW phase to the SDW phase (see Fig. 6), and one obtains a maximum stiffness in the transition regime.

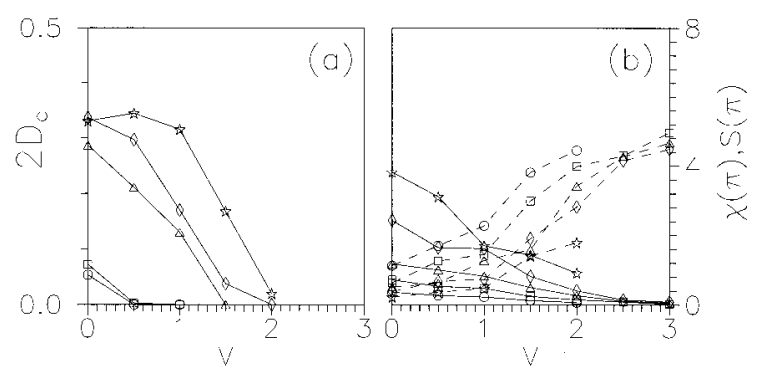

FIG. 5. The same plot as Fig. 2, except that $W=3$. 


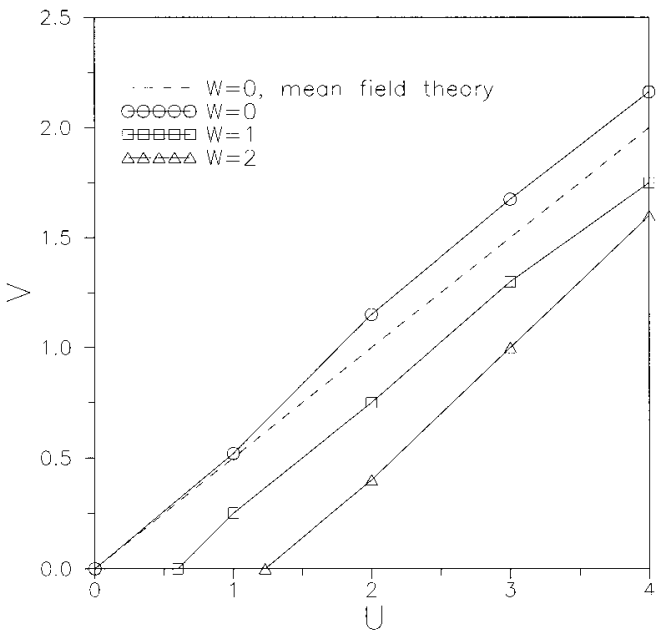

FIG. 6. A schematic plot of the phase-transition lines for the rings at fixed values of disorder strengths. The dashed line is the mean field transition line in clean rings.

We can qualitatively understand the suppression of the charge stiffness at both sides of the transition line by employing a droplet argument envisioned by Hirsch. ${ }^{13}$ In the clean limit, right at the transition line, the CDW phase can tunnel freely to the SDW phase by the formation of droplets of SDW state, and vice versa. The droplet size is arbitrary at $U<U_{c} \approx 3$, where the transition is of second order, and has to be larger than a critical size $n_{\text {crit }}$ at stronger coupling where the transition is of first order. Recalling the checkerboard decomposition, ${ }^{13}$ we note that the strong spontaneous quantum fluctuations on the transition line between the two phases corresponds to the ability to give rise to a large average squared winding number $\left\langle w^{2}\right\rangle$ and thus a large stiffness [see Eq. (3)]. The first-order transition beyond $U=3$ in the clean limit also explains the decreasing peak values of $D_{c}$ beyond $U=3$ in Fig. 2(a). This effect is more prominent in Fig. 7(a), where the stiffness versus the Coulomb interactions is shown for a ring with $L=32$ and at $\beta=L / 2=16$. With increasing departure from the transition line, tunneling to the other phase becomes more and more energetically costly, ${ }^{21}$ i.e., a larger and larger gap ensues for the low-lying excitations, so that we see a decreasing stiffness on both sides of the transition line. From a scaling point of view, we thus expect that $D_{c}$ vanishes beyond $U=3$ in an infinite and clean ring, irrespectively of the nearest-neighbor coupling. This seems to be a pseudoeffect of the Hubbard model, and needs further study. With the help of disorders, it is believable that the droplet size can also be arbitrary in the vicinity of $U=3$, depending on the local realization of the disorders. In other words, the critical point $U_{c}$ would be larger in the presence of disorders. The above scenario is consistent with Figs. 4(a) and 5(a), where the peak value (if it exists) of $D_{c}$ does not decrease with $U$ in the range we considered. To further support the above viewpoint, in Fig. 7(b) we present the stiffness for the same parameters as in Fig. 7(a) except that $W=1$. We see in Fig. 7(b) that the peak value for $U=3$ is compatible with that of $U<3$, in contrast to the situation in Fig. 7(a).

The global amplitude of the stiffness in Fig. 7(b) is close to that in Fig. 4(a). This might suggest that, even in the

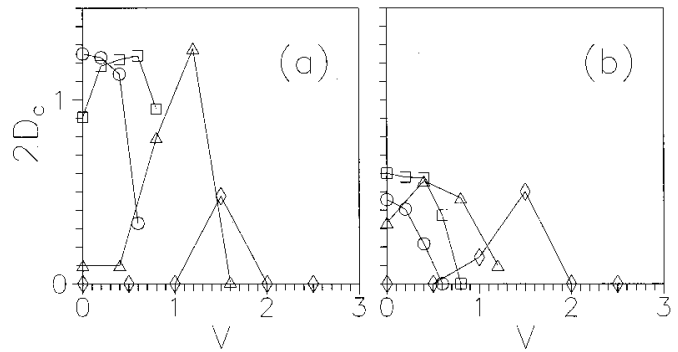

FIG. 7. The stiffness vs $V$ in a 32-site ring and at $\beta=16$ and at various values of on-site repulsion, $U=0$ (circles), $U=1$ (squares), $U=2$ (triangles), and $U=3$ (diamonds). (a) $W=0$. (b) $W=1$.

presence of interactions, the disorder effect enters the stiffness as $L / \xi_{0} \propto L W^{2}$, where $\xi_{0} \approx 105 / W^{2}$ (when $W \ll 2 \pi$ ) is the so-called localization length. ${ }^{5}$ Indeed, from Figs. $2-5$, the global maximum of $D_{c}$ at fixed $W$ agrees approximately with the noninteracting value in disordered systems, i.e., $D_{c, \max }$ $\propto e^{-L / \xi_{0}}$. This indicates that inclusion of Coulomb interactions is still not enough to compensate for the disorder effect, and thus cannot explain the anomalously large current observed in dirty rings. ${ }^{3}$ However, since the reduction of the persistent current by the disorders is most severe for noninteracting fermions, the possibility of the enhancement by the Coulomb interactions discussed in this work is promising, and we expect that the stiffness could be lifted higher by the interactions over the noninteracting limit in disordered rings with lower filling fractions (i.e., $<0.5$ ). Work in this direction has been undertaken.

In closing this section, we would like to point out that our results are limited to the zero-magnetization sector, although the algorithm applies equally well in other sectors as long as the numbers of spin-up and -down electrons have the same parity. Our choice is conventional. It is in fact plausible for noninteracting systems where one would have particle-hole symmetry, but is only approximate for interacting systems. The stiffness may be sensitive to the magnetization. ${ }^{10}$ Unfortunately, the present world-line Monte Carlo method is unable to deal with variable magnetization self-consistently. This may be a drawback of the world-line Monte Carlo method itself. Nevertheless, we expect intuitively that the ground state of an even number of fermions in the ring should be well approximated to lie in the zero-magnetization sector, as long as the Zeeman energy could be ignored, as has been done in this work.

\section{SUMMARY}

We have studied the charge stiffness in disordered halffilled Hubbard rings with electrons interacting via both onsite and nearest-neighbor Coulomb interactions. We have showed that there is an intrinsic relation between the charge stiffness and the magnetic phase of the system: The charge stiffness peaks at the transition line.

\section{ACKNOWLEDGMENT}

We acknowledge the support of an RGC grant of Hong Kong, under Grant No. HKU 262/95P. 
${ }^{1}$ D. Mailly, C. Chapelier, and A. Benoit, Phys. Rev. Lett. 70, 2020 (1993)

${ }^{2}$ M. Büttiker, Y. Imry, and R. Landauer, Phys. Lett. A 96, 365 (1983).

${ }^{3}$ L. P. Levy, G. Dolan, J. Dunsmuir, and H. Bouchiat, Phys. Rev. Lett. 64, 2074 (1990).

${ }^{4}$ V. Chandrasekhar, R. A. Webb, M. J. Brady, M. B. Ketchen, W. J. Gallagher, and A. Kleinsasser, Phys. Rev. Lett. 67, 3578 (1991)

${ }^{5}$ H. Kato and Y. Yoshioka, Phys. Rev. B 50, 4943 (1994).

${ }^{6}$ M. Abraham and R. Berkovits, Phys. Rev. Lett. 70, 1509 (1993).

${ }^{7}$ R. Berkovits and Y. Avishai, Europhys. Lett. 29, 475 (1995).

${ }^{8}$ G. Bouzerar and D. Poilblanc, Phys. Rev. B 52, 10772 (1995).

${ }^{9}$ H. Kato and Y. Yoshioka, Physica B 212, 251 (1995).

${ }^{10}$ R. Römer and A. Punnoose, Phys. Rev. B 52, 14809 (1995).

${ }^{11}$ T. Giamarchi and B. Shastry, Phys. Rev. B 51, 10915 (1995).

${ }^{12}$ M. Ramin, B. Reulet, and H. Bouchiat, Phys. Rev B 51, 5582; M. Kamal, Z. Musslimani, and A. Auerbach, J. Phys. (France) I 5, 1487 (1995).

${ }^{13}$ J. Hirsch, Phys. Rev. Lett. 53, 2327 (1984).

${ }^{14}$ J. Hirsch, D. Scalapino, R. Sugar, and R. Blanckenbecler, Phys.
Rev. B 26, 5033 (1982).

${ }^{15}$ G. Batrouni and R. Scalettar, Phys. Rev. B 46, 9051 (1992).

${ }^{16}$ E. Pollock and D. Ceperley, Phys. Rev. B 36, 8343 (1987).

${ }^{17}$ Q. Wang, J.-X. Zhu, and Z. D. Wang J. Phys. Condens Matter 8, L413 (1996).

${ }^{18}$ Operationally, one can view $\widetilde{Z}$ as the bosonized untwisted partition function except that the Pauli exclusion principle is retained.

${ }^{19}$ H. Mori, Phys. Rev. B 51, 12943 (1995).

${ }^{20}$ In addition, it can be shown that the so-called spin stiffness $D_{s}$ is proportional to $\left\langle\left(w_{\uparrow}-w_{\downarrow}\right)^{2}\right\rangle$, and thus can also be calculated from the zero-frequency limit of the Fourier transform of a similar correlation function $\langle\mathcal{L}(0) \mathcal{L}(\tau)\rangle$, with $\mathcal{L}(\tau)$ $=\Sigma_{i, \sigma} R_{i} \operatorname{sgn}(\sigma)\left[n_{i, \sigma}(\tau+\Delta \tau)-n_{i, \sigma}(\tau)\right]$ where $\operatorname{sgn}()$ is the sign function for the up- and down-spins. For a discussion of $D_{s}$, see, e.g., Ref. 10.

${ }^{21}$ In the absence of impurities, and in a mean-field treatment that neglects the kinetic-energy lowering, the CDW (SDW) droplet of size $n$ nucleating from a pure SDW (CDW) state is $E_{n}=V \pm n(U-2 V)$, where $V$ is the surface energy. 